\title{
Spin scattering and noncollinear spin structure-induced intrinsic anomalous Hall effect in antiferromagnetic topological insulator $\mathrm{MnBi}_{2} \mathrm{Te}_{4}$
}

\author{
Seng Huat Lee $\odot,{ }^{1,2, *}$ Yanglin Zhu, ${ }^{2,3, *}$ Yu Wang,,${ }^{1,2}$ Leixin Miao, ${ }^{4}$ Timothy Pillsbury, ${ }^{2}$ Hemian $\mathrm{Yi}^{2}$ Susan Kempinger, ${ }^{2}$ \\ Jin Hu, ${ }^{5}$ Colin A. Heikes, ${ }^{6}$ P. Quarterman, ${ }^{6}$ William Ratcliff, ${ }^{6}$ Julie A. Borchers, ${ }^{6}$ Heda Zhang, ${ }^{7}$ Xianglin Ke, ${ }^{7}$ David Graf, \\ Nasim Alem, ${ }^{4}$ Cui-Zu Chang, ${ }^{1,2}$ Nitin Samarth,,${ }^{1,2}$ and Zhiqiang Mao ${ }^{1,2, \dagger}$ \\ ${ }^{1} 2$ D Crystal Consortium, Materials Research Institute, Pennsylvania State University, University Park, Pennsylvania 16802, USA \\ ${ }^{2}$ Department of Physics, Pennsylvania State University, University Park, Pennsylvania 16802, USA \\ ${ }^{3}$ Department of Physics and Engineering Physics, Tulane University, New Orleans, Louisiana 70118, USA \\ ${ }^{4}$ Department of Materials Science and Engineering, Pennsylvania State University, University Park, Pennsylvania 16802, USA \\ ${ }^{5}$ Department of Physics, University of Arkansas, Fayetteville, Arkansas 72701, USA \\ ${ }^{6}$ NIST Center for Neutron Research, National Institute of Standards and Technology, Gaithersburg, Maryland 20899, USA \\ ${ }^{7}$ Department of Physics and Astronomy, Michigan State University, East Lansing, Michigan 48824, USA \\ ${ }^{8}$ National High Magnetic Field Laboratory, Tallahassee, Florida 32310, USA
}

(Received 11 December 2018; revised manuscript received 5 May 2019; published 19 August 2019)

\begin{abstract}
$\mathrm{MnBi}_{2} \mathrm{Te}_{4}$ has recently been established as an intrinsic antiferromagnetic (AFM) topological insulatoran ideal platform to create quantum anomalous Hall insulator and axion insulator states. We performed comprehensive studies on the structure, nontrivial surface state, and magnetotransport properties of this material. Our results reveal an intrinsic anomalous Hall effect arising from a noncollinear spin structure for the magnetic field parallel to the $c$ axis. We observed negative magnetoresistance under arbitrary field orientation below and above the Néel temperature $\left(T_{\mathrm{N}}\right)$, providing clear evidence for strong spin fluctuation-driven spin scattering in both the AFM and paramagnetic states. Furthermore, we found that the nontrivial surface state opens a large gap ( $\sim 85 \mathrm{meV}$ ) even far above $T_{\mathrm{N}}$. Our findings demonstrate that the bulk band structure of $\mathrm{MnBi}_{2} \mathrm{Te}_{4}$ is strongly coupled with the magnetic property and that a net Berry curvature in momentum space can be created in the canted AFM state. In addition, our results imply that the gap opening in the surface states is intrinsic, likely caused by the strong spin fluctuations in this material.
\end{abstract}

DOI: 10.1103/PhysRevResearch.1.012011

The recently discovered quantum anomalous Hall (QAH) effect [1-7] is a variant of the Chern insulator first envisioned by Haldane [8]. Characterized by a quantized Hall conductance and vanishing longitudinal conductance at zero magnetic field, the QAH requires combining topology with magnetism $[2,6,7]$. This can be achieved in three ways: magnetic doping of a topological insulator (TI) [3,4], proximity of a TI to a ferromagnetic (FM) or an antiferromagnetic (AFM) insulator [9-15], or in TIs that are intrinsically FM or AFM [16]. The true QAH effect has only been seen in thin TI films of $\mathrm{Cr}$ - and/or V-doped (Bi, Sb) $\mathrm{Te}_{3}$ [3,4]. However, the "critical temperature" required is below $\sim 2 \mathrm{~K}[3,4,17,18]$, severely constraining the exploration of fundamental physics and technological applications. The recent discovery of an intrinsic AFM TI, $\mathrm{MnBi}_{2} \mathrm{Te}_{4}$ [19-21], predicted to show a high-temperature QAH effect in thin films is a key advance in this context.

\footnotetext{
*These authors contributed equally to this work.

†zim1@psu.edu

Published by the American Physical Society under the terms of the Creative Commons Attribution 4.0 International license. Further distribution of this work must maintain attribution to the author(s) and the published article's title, journal citation, and DOI.
}

$\mathrm{MnBi}_{2} \mathrm{Te}_{4}$ crystallizes in a rhombohedral structure with the space group $R \overline{3} m$ [22], built of the stacking of Te$\mathrm{Bi}-\mathrm{Te}-\mathrm{Mn}-\mathrm{Te}-\mathrm{Bi}-\mathrm{Te}$ septuple layers (SLs) along the $c$ axis [inset of Fig. 1(b)]. The antiferromagnetism of this material is produced by the Mn sublattice, while its nontrivial surface state is formed by inverted $\mathrm{Bi}$ and $\mathrm{Te} p_{z}$ bands at the $\Gamma$ point due to strong spin-orbital coupling (SOC). Since its AFM state is characterized by an $A$-type AFM order $\left(T_{\mathrm{N}}=25 \mathrm{~K}\right)$ [21], formed by the Mn FM layers stacked antiferromagnetically along the $c$ axis [21,23], the FM layer near the cleavage surface is anticipated to break timereversal symmetry (TRS), thus opening a large gap ( $\sim 50$ or $88 \mathrm{meV}[20,21,24]$ ) in the topological surface state; this has been probed in angle-resolved photoemission spectroscopy (ARPES) measurements on single-crystal samples [21,24].

$\mathrm{MnBi}_{2} \mathrm{Te}_{4}$ is expected to be an ideal quantum material for hosting interesting topological phases, including a hightemperature QAH insulator in thin films with odd numbers of SLs [19,20,25], an axion insulator state [9,19] exhibiting the topological magnetoelectric effect in thin films with even numbers of SLs, an ideal Weyl semimetal state with a single pair of Weyl nodes near the Fermi level [19,20], and possibly chiral Majorana modes via interaction with an $s$-wave superconductor [26]. Recently, both the magnetic-field-driven quantized Hall state (Chern insulator) and the axion insulator at zero field have been observed in exfoliated $\mathrm{MnBi}_{2} \mathrm{Te}_{4}$ 
(a)

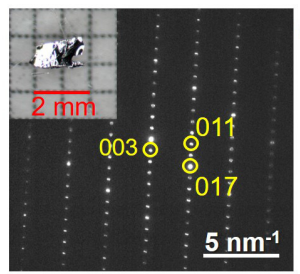

(b)
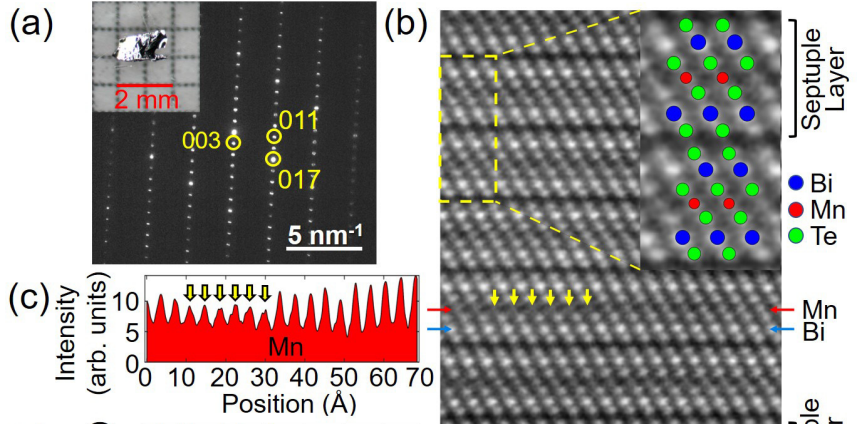

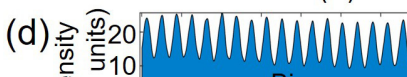

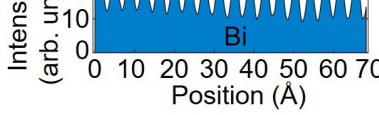

FIG. 1. (a) The selected area diffraction pattern taken from the [100] zone axis for $\mathrm{MnBi}_{2} \mathrm{Te}_{4}$. Inset: Crystal image of $\mathrm{MnBi}_{2} \mathrm{Te}_{4}$. (b) HAADF-STEM image taken from the [100] zone axis. Inset: Magnified image of a one-unit cell with the atoms overlaid on top to demonstrate the layered structure of $\mathrm{MnBi}_{2} \mathrm{Te}_{4}$. The yellow arrows in (b) mark Mn vacancies. (c) and (d) The intensity profiles of Mn and $\mathrm{Bi}$ atomic columns indicated by the red and blue arrows in (b).

thin films [27,28], which makes this material a fascinating playground for the study of topological quantum states.

In this Rapid Communication, we report a systematic study of single-crystal samples of $\mathrm{MnBi}_{2} \mathrm{Te}_{4}$, focusing on elucidating the coupling between bulk electronic and magnetic properties, as well as exploring how surface states are related to magnetism. Our studies reveal clear evidence of spin fluctuation-driven spin scattering in both the AFM ordered state and the paramagnetic (PM) state above $T_{\mathrm{N}}$, suggesting that the opening of a gap in the nontrivial surface states in the PM state is likely driven by the strong spin fluctuations in this material. Moreover, we observed an intrinsic anomalous Hall effect not linearly coupled with magnetization in a canted AFM phase for the fields applied along the $c$ axis, indicating the presence of a net Berry curvature in momentum space created by the noncollinear spin structure. These findings would advance understanding of the underlying physics of this exciting material.

$\mathrm{MnBi}_{2} \mathrm{Te}_{4}$ single crystals [inset of Fig. 1(a)] were synthesized using the melt growth method [21] [see the Supplemental Material (SM) [29] for details]. The structure of $\mathrm{MnBi}_{2} \mathrm{Te}_{4}$ crystals were analyzed using x-ray diffraction (Fig. S1) and high-resolution scanning transmission electron microscope (STEM) imaging. The dotlike diffraction spots seen in the electron diffraction pattern taken along the [100] zone axis [Fig. 1(a)] indicate the single crystallinity of the sample with no stacking faults between SLs. The atomic resolution high angle annular dark field (HAADF)-STEM image taken along the [100] zone axis clearly demonstrates the layered structure of $\mathrm{MnBi}_{2} \mathrm{Te}_{4}$ comprising the stacking of Te-Bi-Te-Mn-Te-BiTe SLs along the $c$ axis [Fig. 1(b)] [22]. Figures 1(c) and 1(d) show the uniform intensity line profile of $\mathrm{Mn}$ and $\mathrm{Bi}$ atoms, delineated by red and blue arrows in the STEM image, indicating little to no intermixing of the two elements. Additionally, within the Mn intensity line profile, peaks with lower Mn intensity can be seen (marked by yellow arrows), indicating
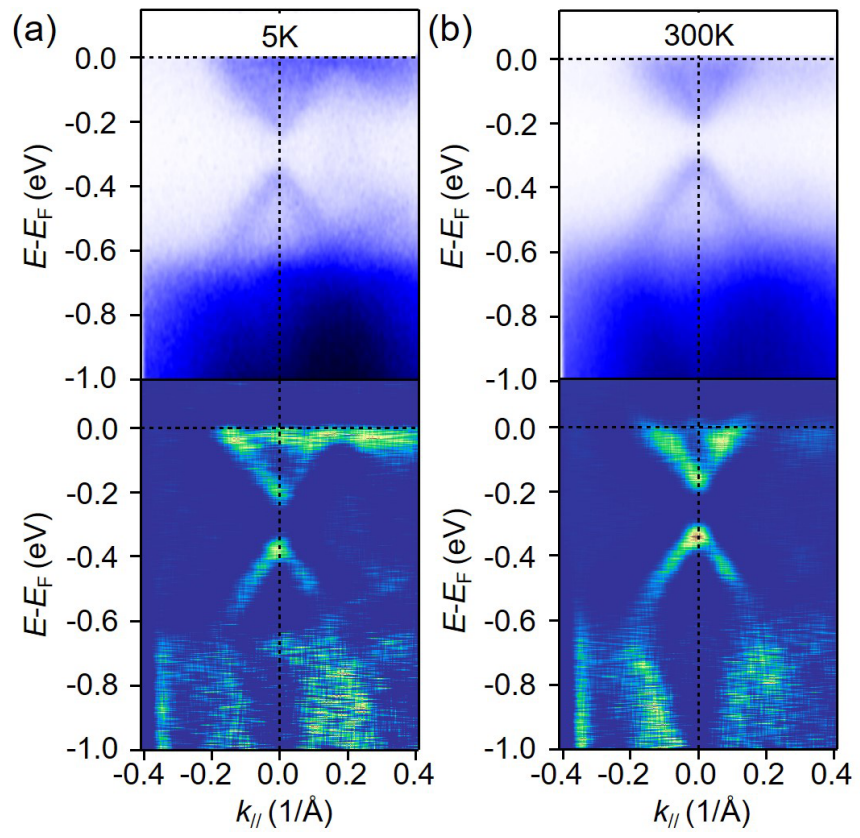

FIG. 2. ARPES band maps of $\mathrm{MnBi}_{2} \mathrm{Te}_{4}$ single crystal along the $K-\Gamma-K$ direction and the corresponding second derivative maps at (a) $5 \mathrm{~K}$ and (b) $300 \mathrm{~K}$.

Mn vacancies within the atomic column. Mn vacancies can also be distinguished in the bright field (BF) and low angle annular dark field (LAADF)-STEM images (Fig. S2) and the resulting strain contrast associated with them.

A key signature of the AFM TI state in $\mathrm{MnBi}_{2} \mathrm{Te}_{4}$ is that its surface with preserved $S$ symmetry has a gapless Dirac cone, while the surface with broken $S$ symmetry is characterized by a Dirac cone with a large gap (50 or $88 \mathrm{meV}[20,21]) . S$ stands for a combined symmetry, defined as $S=\Theta T_{1 / 2}$, where $\Theta$ represents TRS and $T_{1 / 2}$ denotes primitive-lattice transition symmetry [30]. The (001) surface, the cleavage plane, is an $S$-symmetry broken surface in the AFM state and should thus have a gapped Dirac cone [20]. Such a gapped Dirac cone has recently been probed in the ARPES band maps of singlecrystal samples [21,24]; the gap magnitude measured at the $\Gamma$ point is $\sim 70 \mathrm{meV}$ at $17 \mathrm{~K}$, consistent with the theoretically calculated gap $[19,20]$. We also performed ARPES measurements on our $\mathrm{MnBi}_{2} \mathrm{Te}_{4}$ single-crystal samples and observed clear signatures of gapped surface states at both 5 and $300 \mathrm{~K}$, as shown in Figs. 2(a) and 2(b). The gap magnitude is $\sim 85 \mathrm{meV}$ at both temperatures, consistent with the previous ARPES studies [21,24]. This observation indicates that the gap opening observed here does not originate from the AFM order in $\mathrm{MnBi}_{2} \mathrm{Te}_{4}$. We speculate such a surface state gap opening in $\mathrm{MnBi}_{2} \mathrm{Te}_{4}$ is driven by spin fluctuations in the $\mathrm{PM}$ phase, as discussed below.

Using magnetic susceptibility measurements on $\mathrm{MnBi}_{2} \mathrm{Te}_{4}$ [Fig. S3(a)], we observed an AFM transition at $\sim 25 \mathrm{~K}$ with the spin-easy axis along the $c$ axis. The isothermal magnetization measurements for $H \| c$ [Figs. 3(a) and S3(b)] reveal a remarkable spin-flop transition near $3.57 \mathrm{~T}$ at $2 \mathrm{~K}$ (denoted by $H_{c 1}$ hereafter), which leads the system to a metastable canted AFM (CAFM) state. The noncollinear spin structure of the CAFM phase is verified by neutron scattering measurements, 

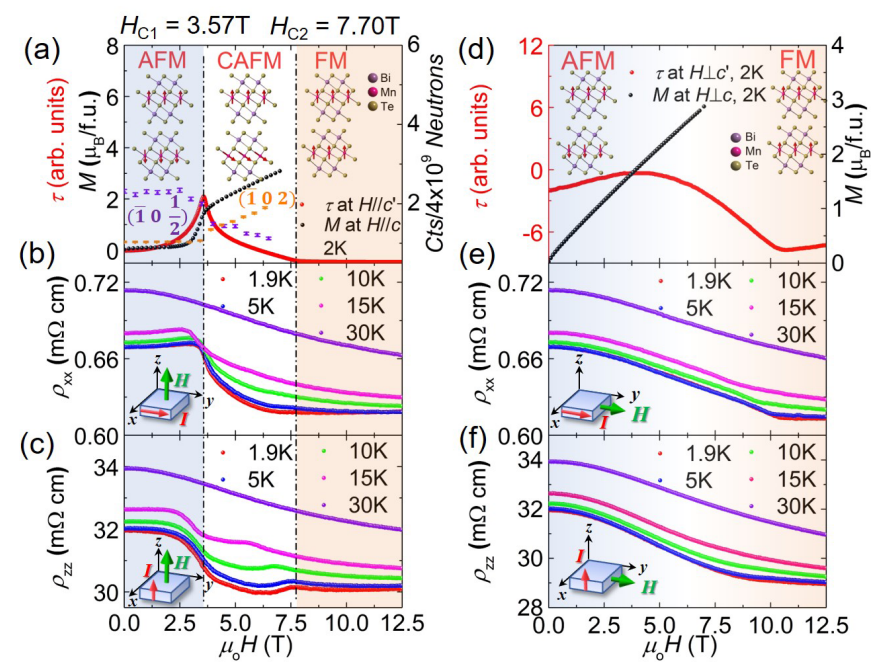

FIG. 3. (a) and (d): Field dependence of magnetic torque $\tau$ of $\mathrm{MnBi}_{2} \mathrm{Te}_{4}$ at $2 \mathrm{~K}$ for (a) $H \| c^{\prime}$ and (d) $H \perp c^{\prime}$ ( $c^{\prime}$ denotes a direction nearly parallel to the $c$ axis). The purple and orange data points in (a) are the neutron scattering intensities at $\left(\overline{1} 0 \frac{1}{2}\right)$ and (102) as a function of magnetic field at $4.2 \mathrm{~K}$. Error bars represent one sigma errors based on Poisson counting statistics. The black curves in (a) and (d) represent the isothermal magnetization measured at $2 \mathrm{~K}$ with $H \| c$ and $H \perp c$. The insets illustrate the antiferromagnetic (AFM), canted antiferromagnetic (CAFM), and ferromagnetic (FM) phases in the different field ranges. (b) and (c): Field dependences of in-plane resistivity $\rho_{x x}$ and out-of-plane resistivity $\rho_{z z}$ at various temperatures for $H \| c$. (e) and (f): Field dependences of $\rho_{x x}$ and $\rho_{z z}$ at various temperatures for $H \perp c$. The insets in (b), (c), (e), and (f) illustrate the experiment setups.

which finds the AFM reflection peak ( $\left.10 \frac{1}{2}\right)$ intensity decreases at magnetic fields above $3 \mathrm{~T}$, while the $\mathrm{FM}$ diffraction peak (102) intensity increases, with an inflection point near $3.5 \mathrm{~T}$ (see Sec. 5 in SM for details). Moreover, we also conducted magnetic torque measurements and found the transition from the CAFM to FM phase occurs at $\sim 7.70 \mathrm{~T}$ (denoted by $H_{c 2}$ ), as shown in Fig. 3(a). The measured torque is not directly proportional to magnetization, but dependent on the components of the magnetization and magnetic field along the $c$ axis and in-plane directions of the sample, as explained in the SM (Sec. 6). In Fig. 3(a), we also include schematics of three distinct magnetic phases (i.e., AFM, CAFM, and FM) in the different field ranges. However, the metamagnetic transition does not appear for $H \perp c$. The isothermal magnetization measured at $2 \mathrm{~K}$ for $H \perp c$ displays a linear field dependence [Fig. 3(d)], indicating that the AFM state is gradually polarized to a FM state. The high-field torque [Fig. 3(d)] and magnetotransport measurements [Figs. 3(e) and 3(f)] indicate the full FM polarization occurs around $10.5 \mathrm{~T}$.

Figures 4(a) and 4(b) present the temperature dependences of the in-plane $\left(\rho_{x x}\right)$ and out-of-plane $\left(\rho_{z z}\right)$ resistivity under different magnetic fields $(H \| c)$. Both $\rho_{x x}(T)$ and $\rho_{z z}(T)$ at zero field exhibit metallic behavior above $40 \mathrm{~K}$ with a moderate electronic anisotropy $\left(\rho_{z z} / \rho_{x x} \sim 35\right.$ at $\left.2 \mathrm{~K}\right)$ and remarkable peaks at the AFM transition. Such resistivity peaks near $T_{\mathrm{N}}$ can be well understood in terms of spin scattering due to spin fluctuations as discussed below. In general, in AFM
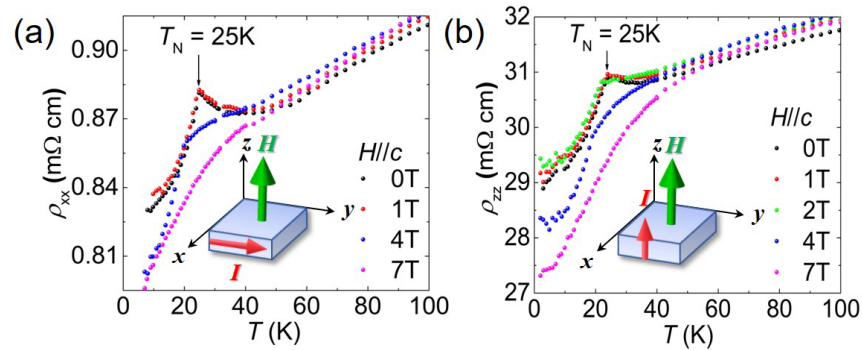

FIG. 4. Temperature dependences of the (a) in-plane resistivity $\rho_{x x}$ and (b) out-of-plane resistivity $\rho_{z z}$ under various magnetic fields $(H \| c)$.

or FM materials, spin fluctuations always tend to intensify as the temperature approaches the ordering temperature where they reach maximum strength. For a local-moment AFM state, the intensified spin fluctuations due to spin-wave excitations near $T_{\mathrm{N}}$ can greatly affect transport properties through spin scattering if itinerant carriers interact with local moments. This results in a resistivity peak near $T_{\mathrm{N}}$. Such a scenario was previously demonstrated in an AFM material $\mathrm{Fe}_{1+y} \mathrm{Te}$ [31]. The resistivity peak near $T_{\mathrm{N}}$ observed here should follow a similar mechanism.

We systematically measured the variation of resistivity under an external magnetic field. Figures 4(a) and 4(b) show that the peaks near $T_{\mathrm{N}}$ in both $\rho_{x x}(T)$ and $\rho_{z z}(T)$ are suppressed by the magnetic fields higher than $H_{c 1}$. This observation is consistent with the spin fluctuation-driven spin-scattering scenario. Further, for an $A$-type AFM system, interlayer AFM coupling usually generates strong spin scattering in the ordered state, resulting in a high-resistivity state. However, when the applied field is strong enough to overcome the interlayer AFM coupling and push it to a forced FM state, the spin scattering would be suppressed, resulting in a lowresistivity state. Such a phenomenon is termed as a spin-valve effect and was first demonstrated in magnetic, multilayer thin films [32,33]. The spin-valve effect can also occur between an $A$-type AFM and a CAFM state as seen in $\mathrm{Ca}_{3} \mathrm{Ru}_{2} \mathrm{O}_{7}$ [34]. We indeed observed a similar spin-valve effect in the magnetotransport measurements of $\mathrm{MnBi}_{2} \mathrm{Te}_{4}$, as shown below.

We performed magnetic field sweep measurements of both $\rho_{x x}$ and $\rho_{z z}$ at various temperatures for both $H \| c$ and $H \perp c$, as presented in Fig. 3. For $H \| c, \rho_{x x}(H)$ and $\rho_{z z}(H)$ show a steep decrease in response to the AFM-toCAFM transition at $H_{c 1}$ [Figs. 3(b) and 3(c)], consistent with the expected spin-valve behavior, indicating the interlayer AFM ordering results in strong spin scattering. When the system is across the CAFM-to-FM transition at $H_{c 2}, \rho_{x x}(H)$ is constant, while $\rho_{z z}(H)$ displays a little hump at $H_{c 2}$ which shifts to lower field at higher temperatures. These observations suggest that the spin scattering due to the interlayer AFM coupling is mostly suppressed in the CAFM phase. The little hump near $H_{c 2}$ observed in $\rho_{z z}(H)$ is likely due to the fact that the magnetoresistivity also contains a positive term induced by the Lorentz effect and anisotropic Fermi surface, as evidenced by the data measured above $15 \mathrm{~T}$ (Fig. S7).

The suppression of spin scattering by magnetic field is also observed in $\rho_{x x}(H)$ and $\rho_{z z}(H)$ measurements for $H \perp c$ 

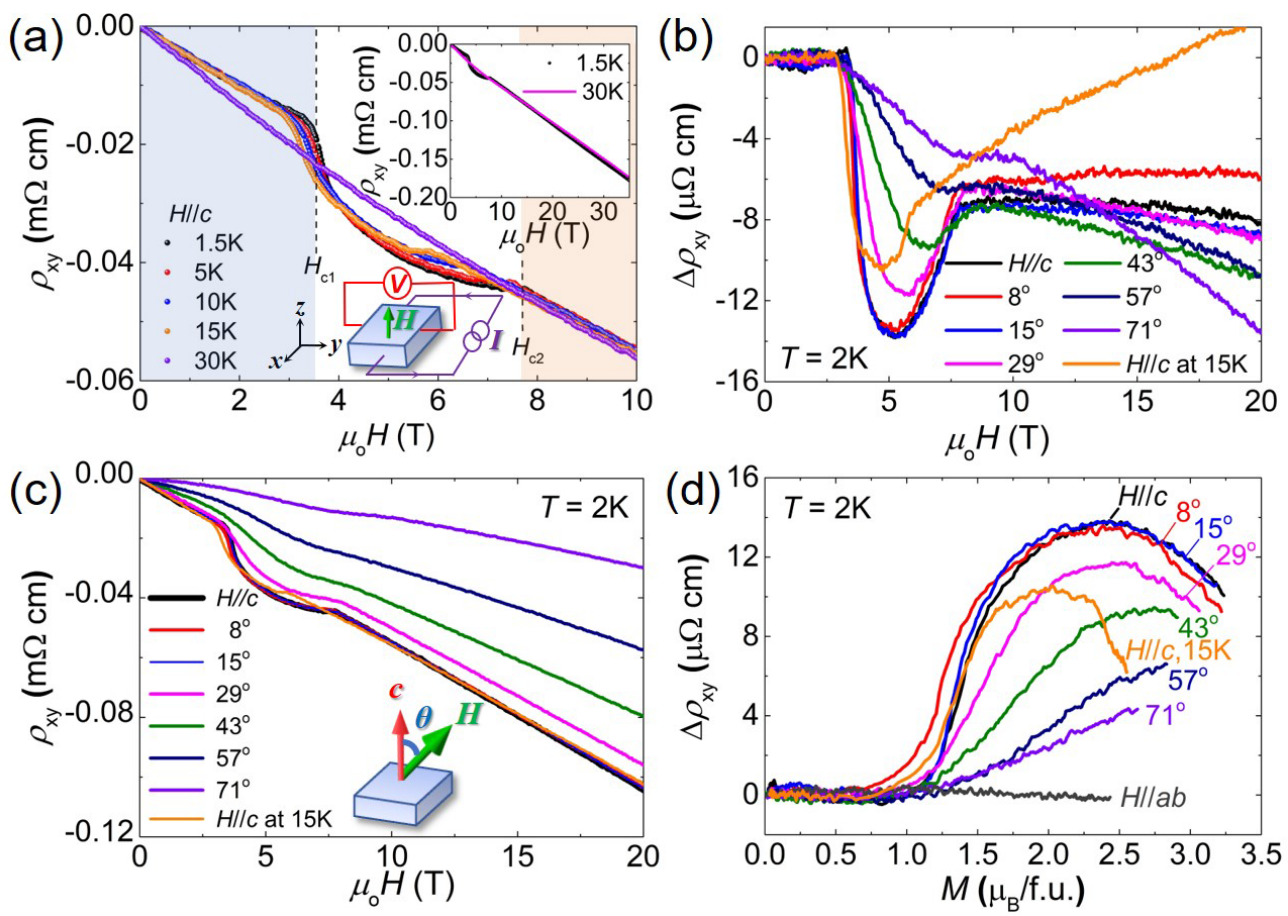

FIG. 5. (a) Hall resistivity $\rho_{x y}$ as a function of magnetic field at various temperatures for $\mathrm{MnBi}_{2} \mathrm{Te}_{4}$. The schematic illustrates the experimental setup for $\rho_{x y}$ measurements. Inset: $\rho_{x y}$ vs $H$ in the $0-35 \mathrm{~T}$ field range at 1.5 and $30 \mathrm{~K}$. (b) The field dependence of anomalous Hall resistivity $\Delta \rho_{x y}$ under various field orientations, obtained after subtracting the normal Hall resistivity due to the Lorentz effect from $\rho_{x y}$ shown in (c). (c) Hall resistivity as a function of magnetic field under various field orientations. (d) $\Delta \rho_{x y}$ plotted against the magnetization $M$ for different field orientations. The data in orange color were collected at $15 \mathrm{~K}$ and the rest were measured at $2 \mathrm{~K}$ [the magnetization data used for making this plot are shown in Fig. S3(c)].

[Figs. 3(e) and 3(f)]. Since the AFM phase is gradually polarized to a FM state in this field configuration [Fig. 3(d)], $\rho_{x x}(H)$ and $\rho_{z z}(H)$ show a gradual decrease with increasing field and then tend to level off near $H_{c 2}$. In addition to observing spin scattering in the AFM state, we also found spin scattering is substantial in the PM state, as evidenced by the large negative magnetoresistance (MR) at $T=30 \mathrm{~K}$ for both $H \| c$ and $H \perp c$ [e.g., $\left[\rho_{z z}(H)-\rho_{z z}(0)\right] / \rho_{z z}(0) \sim-6 \%$ to $-9 \%$ at $12.5 \mathrm{~T}$ and $30 \mathrm{~K}$, comparable to the magnitude of negative MR seen in the AFM state in the same field range]. These results strongly support that the PM state has strong spin fluctuations. Since the intralayer Mn-Te-Mn FM superexchange interactions are much stronger than the interlayer AFM interaction, the spin fluctuations in the PM state should feature FM correlation (i.e., FM spin fluctuations), as manifested in the positive Curie-Weiss temperature $\left(\theta_{\mathrm{CW}} \sim\right.$ $5 \mathrm{~K}$ ) extracted from the susceptibility [inset of Fig. S3(a)]. Such strong FM fluctuations might break TRS symmetry, thus resulting in the gap opening of surface states as observed in ARPES experiments (Fig. 2). The spin fluctuation-driven gap opening in the nontrivial surface state in magnetic doped TIs has indeed been established in previous work [35]. We note the gap magnitude of the surface states hardly varies with temperature (Fig. 2), which is consistent with previous reports $[21,24]$ and can possibly be attributed to the strong spin fluctuations arising from intralayer Mn-Te-Mn FM superexchange interactions.

Another possible consequence of strong spin scattering is low transport mobility. This is also verified in our Hall resistivity $\rho_{x y}$ measurements. Figure 5(a) shows $\rho_{x y}$ as a function of magnetic field at various temperatures. From these data, we found the carrier mobility is indeed low. However, we observed an intrinsic anomalous Hall (AH) effect in the CAFM phase. When the field is increased above $H_{c 2}$, all the data taken at different temperatures collapse onto a single line up to the maximum measurement field $35 \mathrm{~T}$ [inset of Fig. 5(a)], consistent with the single-band nature of this system. The Hall coefficient $R_{\mathrm{o}}$ extracted from such a linear field dependence of $\rho_{x y}$ is $-4.76 \times 10^{-10} \Omega \mathrm{cm} / \mathrm{Oe}$, from which the carrier mobility $\mu\left(=R_{\mathrm{o}} / \rho_{x x}\right)$ is estimated to $79 \mathrm{~cm}^{2} / \mathrm{V} \mathrm{s}$ at $30 \mathrm{~K}$, much less than the carrier mobility in bulk $\mathrm{Bi}_{2} \mathrm{Te}_{3}$ $\left(>800 \mathrm{~cm}^{2} / \mathrm{V} \mathrm{s}\right)[36,37]$. The carrier density estimated from $R_{\mathrm{o}}$ is $1.31 \times 10^{20} \mathrm{~cm}^{-3}$, falling into the carrier density range of $\mathrm{Bi}_{2} \mathrm{Te}_{3}\left(10^{17}-10^{20} \mathrm{~cm}^{-3}\right)$ [37,38]. The negative sign of $R_{\mathrm{o}}$ indicates that the carriers in our samples are electron type, consistent with the ARPES measurement results (Fig. 2).

Next, we focus on the intrinsic AH effect observed in the CAFM phase. The $\rho_{x y}$ of this phase shows striking humplike features for $H \| c$, contrasted with the linear field dependence in the AFM phase below $H_{c 1}$ and the FM phase above $H_{c 2}$ [Fig. 5(a)]. The anomalous hump is gradually suppressed as the field is tilted toward the in-plane direction [Fig. 5(c)]. Since the linear increase of $\rho_{x y}$ above $H_{c 2}$ is due to the normal Hall resistivity $\rho_{x y}^{N}$, we obtain the AH resistivity $\Delta \rho_{x y}$ after subtracting $\rho_{x y}^{N}$, as shown in Fig. 5(b), where $\Delta \rho_{x y}$ under various field orientations is also presented. $\Delta \rho_{x y}$ can be further expressed as $\Delta \rho_{x y}=\rho_{x y}^{A}+\rho_{x y}^{T}$ [39], where $\rho_{x y}^{A}$ represents the AH resistivity linearly coupled to the magnetization, whereas 
$\rho_{x y}^{T}$ stands for the AH resistivity due to the net Berry curvature in the momentum space caused by the noncollinear spin structure. $\rho_{x y}^{A}$ arises from the combination of the extrinsic (the skew scattering and the side jump) and intrinsic contributions and is proportional to magnetization [40]. To separate $\rho_{x y}^{T}$ from $\Delta \rho_{x y}$, we plot $\Delta \rho_{x y}$ as a function of magnetization $M$ in Fig. 5(d) [note the magnetization data under various field orientations are shown in Fig. S3(c)]. We find $\Delta \rho_{x y}(M)$ is nearly linear for the AFM phase below $H_{c 1}$, but exhibits significant humps due to the presence of $\rho_{x y}^{T}$ in the CAFM phase [Fig. 5(d)]. The maximum $\rho_{x y}^{T}$ for $H \| c$ reaches $\sim 14 \mu \Omega \mathrm{cm}$, more than twice that seen in the chiral antiferromagnet $\mathrm{Mn}_{3} \mathrm{Sn}$ [41]. The angular dependence of $\Delta \rho_{x y}(M)$ in Fig. 5(d) shows $\rho_{x y}^{T}$ is strongly suppressed for $\theta>50^{\circ}$. Additionally, we also analyzed $\Delta \rho_{x y}(M)$ of $H \| c$ at $15 \mathrm{~K}$ where $H_{c 1}$ and $H_{c 2}$ can be directly probed from magnetization [Fig. S3(c)] and find the $\rho_{x y}^{T}$ component of the CAFM phase is also significant at this temperature [Fig. 5(d)].

A large $\rho_{x y}^{T}$ is generally expected for an AFM state with noncollinear spin structure [42]. Symmetry breaking plus strong SOC in such systems can lift spin degeneracy, which can lead to a net Berry curvature in momentum space, thus resulting in an intrinsic $\mathrm{AH}$ effect. As indicated above, the noncollinear spin structure of the CAFM phase is evidenced by both the magnetization and neutron scattering measurements. Therefore, the large $\rho_{x y}^{T}$ value of the CAFM phase can be reasonably attributed to the noncollinear spin structure. This result implies the electronic band structure of $\mathrm{MnBi}_{2} \mathrm{Te}_{4}$ is strongly coupled to its magnetism, offering an opportunity to observe different topological states tuned by a magnetic field. Theoretical studies have shown the AFM TI state can evolve into an ideal Weyl semimetal state when the system transforms into a FM phase under magnetic field. However, we did not observe any transport signatures of a Weyl state in the FM phase (such as chiral anomaly) probably because of the Weyl node far from the Fermi level.

In summary, the strong coupling between electronic and magnetic properties of $\mathrm{MnBi}_{2} \mathrm{Te}_{4}$ provides clear evidence of spin scattering in both the AFM and PM states. We attribute the strong spin scattering in the PM state to spin fluctuations that open a gap in the surface states above $T_{\mathrm{N}}$. Moreover, we find a canted AFM state that exhibits an intrinsic $\mathrm{AH}$ effect as a result of the net Berry curvature in the momentum space induced by the noncollinear spin structure, indicating that the electronic band structure of $\mathrm{MnBi}_{2} \mathrm{Te}_{4}$ is strongly coupled with magnetism. Given this material has been demonstrated to host axion insulator and Chern insulator phases, our findings will be important for further understanding of this fascinating material.

We thank Chaoxing Liu and Jun Zhu for useful discussions. The study is based upon research conducted at The Pennsylvania State University Two-Dimensional Crystal ConsortiumMaterials Innovation Platform (2DCC-MIP) which is supported by NSF Cooperative Agreement No. DMR-1539916. Y.L.Z. is supported by the US Department of Energy under EPSCoR Grant No. DESC0012432 with additional support from the Louisiana Board of Regents. Z.Q.M. acknowledges the support from the US National Science Foundation under Grant No. DMR1707502. C.Z.C. acknowledges the support from the DOE grant (DESC0019064) (ARPES measurements). X.L.K. acknowledges the support from the National Science Foundation under Award No. DMR-1608752. J.H. acknowledges the support from the US Department of Energy (DOE), Office of Science, Basic Energy Sciences program under Award No. DE-SC0019467 for the torque analysis. The work at the National High Magnetic Field Laboratory is supported by the NSF Cooperative Agreement No. DMR1157490 and the State of Florida.
[1] C.-X. Liu, X.-L. Qi, X. Dai, Z. Fang, and S.-C. Zhang, Phys. Rev. Lett. 101, 146802 (2008).

[2] R. Yu, W. Zhang, H. J. Zhang, S. C. Zhang, X. Dai, and Z. Fang, Science 329, 61 (2010).

[3] C. Z. Chang et al., Science 340, 167 (2013).

[4] C. Z. Chang, W. Zhao, D. Y. Kim, H. Zhang, B. A. Assaf, D. Heiman, S.-C. Zhang, C. Liu, M. H. W. Chan, and J. S. Moodera, Nat. Mater. 14, 473 (2015).

[5] C.-X. Liu, S.-C. Zhang, and X.-L. Qi, Annu. Rev. Condens. Matter Phys. 7, 301 (2016).

[6] C.-Z. Chang and M. Li, J. Phys.: Condens.: Matter 28, 123002 (2016).

[7] K. He, Y. Wang, and Q.-K. Xue, Annu. Rev. Condens. Matter Phys. 9, 329 (2018).

[8] F. D. M. Haldane, Phys. Rev. Lett. 61, 2015 (1988).

[9] X.-L. Qi, T. L. Hughes, and S.-C. Zhang, Phys. Rev. B 78, 195424 (2008).

[10] P. Wei, F. Katmis, B. A. Assaf, H. Steinberg, P. Jarillo-Herrero, D. Heiman, and J. S. Moodera, Phys. Rev. Lett. 110, 186807 (2013).
[11] Z. Jiang, C.-Z. Chang, C. Tang, P. Wei, J. S. Moodera, and J. Shi, Nano Lett. 15, 5835 (2015).

[12] F. Katmis et al., Nature (London) 533, 513 (2016).

[13] Z. Jiang, C.-Z. Chang, M. R. Masir, C. Tang, Y. Xu, J. S. Moodera, A. H. MacDonald, and J. Shi, Nat. Commun. 7, 11458 (2016).

[14] H. Wang, J. Kally, J. S. Lee, T. Liu, H. Chang, D. R. Hickey, K. A. Mkhoyan, M. Wu, A. Richardella, and N. Samarth, Phys. Rev. Lett. 117, 076601 (2016).

[15] C. Tang et al., Sci. Adv. 3, e1700307 (2017).

[16] P. Tang, Q. Zhou, G. Xu, and S.-C. Zhang, Nat. Phys. 12, 1100 (2016).

[17] M. Mogi, R. Yoshimi, A. Tsukazaki, K. Yasuda, Y. Kozuka, K. S. Takahashi, M. Kawasaki, and Y. Tokura, Appl. Phys. Lett. 107, 182401 (2015).

[18] Y. B. Ou et al., Adv. Mater. 30, 1703062 (2018).

[19] D. Zhang, M. Shi, T. Zhu, D. Xing, H. Zhang, and J. Wang, Phys. Rev. Lett. 122, 206401 (2019).

[20] J. Li, Y. Li, S. Du, Z. Wang, B.-L. Gu, S.-C. Zhang, K. He, W. Duan, and Y. Xu, Sci. Adv. 5, eaaw5685 (2019). 
[21] M. M. Otrokov et al., arXiv:1809.07389.

[22] D. S. Lee, T.-H. Kim, C.-H. Park, C.-Y. Chung, Y. S. Lim, W.-S. Seo, and H.-H. Park, CrystEngComm 15, 5532 (2013).

[23] J.-Q. Yan, Q. Zhang, T. Heitmann, Z. Huang, K. Y. Chen, J.-G. Cheng, W. Wu, D. Vaknin, B. C. Sales, and R. J. McQueeney, Phys. Rev. Materials 3, 064202 (2019).

[24] R. C. Vidal et al., arXiv:1903.11826.

[25] M. M. Otrokov, I. P. Rusinov, M. Blanco-Rey, M. Hoffmann, A. Yu. Vyazovskaya, S. V. Eremeev, A. Ernst, P. M. Echenique, A. Arnau, and E. V. Chulkov, Phys. Rev. Lett. 122, 107202 (2019).

[26] Y. Peng and Y. Xu, Phys. Rev. B 99, 195431 (2019).

[27] Y. Deng, Y. Yu, M. Zhu Shi, J. Wang, X. H. Chen, and Y. Zhang, arXiv:1904.11468.

[28] C. Liu et al., arXiv:1905.00715.

[29] See Supplemental Material at http://link.aps.org/supplemental/ 10.1103/PhysRevResearch.1.012011 for details of crystal growth, scanning transmission electron microscope, ARPES, magnetization, neutron diffraction, high-magnetic field magnetotransport measurement and torque simulations.

[30] R. S. K. Mong, A. M. Essin, and J. E. Moore, Phys. Rev. B 81, 245209 (2010).

[31] T. J. Liu et al., Phys. Rev. B 80, 174509 (2009).
[32] M. N. Baibich, J. M. Broto, A. Fert, F. N. Vandau, F. Petroff, P. Eitenne, G. Creuzet, A. Friederich, and J. Chazelas, Phys. Rev. Lett. 61, 2472 (1988).

[33] G. Binasch, P. Grunberg, F. Saurenbach, and W. Zinn, Phys. Rev. B 39, 4828 (1989).

[34] M. Zhu, T. Hong, J. Peng, T. Zou, Z. Q. Mao, and X. Ke, J. Phys.: Condens. Matter 30, 075802 (2018).

[35] C. Z. Chang et al., Phys. Rev. Lett. 112, 056801 (2014).

[36] D.-X. Qu, Y. S. Hor, J. Xiong, R. J. Cava, and N. P. Ong, Science 329, 821 (2010).

[37] K. Shrestha, M. Chou, D. Graf, H. D. Yang, B. Lorenz, and C. W. Chu, Phys. Rev. B 95, 195113 (2017).

[38] J. P. Fleurial, L. Gailliard, R. Triboulet, H. Scherrer, and S. Scherrer, J. Phys. Chem. Solids 49, 1237 (1988).

[39] T. Suzuki, R. Chisnell, A. Devarakonda, Y. T. Liu, W. Feng, D. Xiao, J. W. Lynn, and J. G. Checkelsky, Nat. Phys. 12, 1119 (2016).

[40] N. Nagaosa, J. Sinova, S. Onoda, A. H. MacDonald, and N. P. Ong, Rev. Mod. Phys. 82, 1539 (2010).

[41] S. Nakatsuji, N. Kiyohara, and T. Higo, Nature (London) 527, 212 (2015).

[42] H. Chen, Q. Niu, and A. H. MacDonald, Phys. Rev. Lett. 112 , 017205 (2014). 